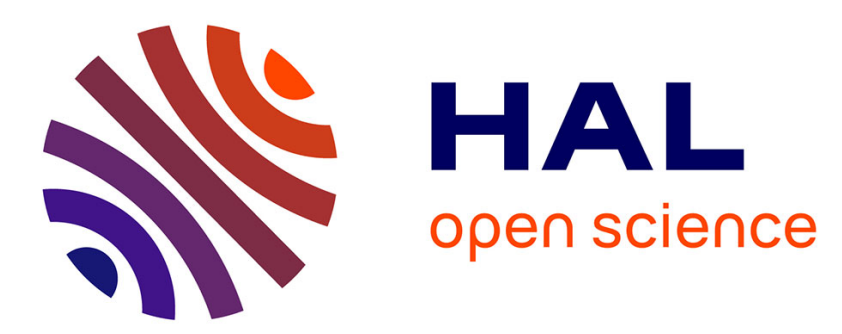

\title{
Gas-induced fluidization of mobile liquid-saturated grains
}

Gabriel Ramos, Germán Varas, Jean-Christophe Géminard, Valérie Vidal

\section{To cite this version:}

Gabriel Ramos, Germán Varas, Jean-Christophe Géminard, Valérie Vidal. Gas-induced fluidization of mobile liquid-saturated grains. Physical Review E: Statistical, Nonlinear, and Soft Matter Physics, 2015, 10.1103/PhysRevE.92.062210 . hal-01249898

\section{HAL Id: hal-01249898 \\ https://hal.science/hal-01249898}

Submitted on 4 Jan 2016

HAL is a multi-disciplinary open access archive for the deposit and dissemination of scientific research documents, whether they are published or not. The documents may come from teaching and research institutions in France or abroad, or from public or private research centers.
L'archive ouverte pluridisciplinaire HAL, est destinée au dépôt et à la diffusion de documents scientifiques de niveau recherche, publiés ou non, émanant des établissements d'enseignement et de recherche français ou étrangers, des laboratoires publics ou privés. 


\title{
Gas-induced fluidization of mobile liquid-saturated grains
}

\author{
Gabriel Ramos, ${ }^{1}$ Germán Varas, ${ }^{1}$ Jean-Christophe Géminard, ${ }^{2}$ and Valérie Vidal ${ }^{2}$ \\ ${ }^{1}$ Instituto de Fisica, Pontificia Universidad Católica de Valparaiso, Av. Universidad 330, Valparaiso, Chile \\ ${ }^{2}$ Laboratoire de Physique, École Normale Supérieure de Lyon, Université de Lyon, CNRS UMR5672, 46 Allée d'Italie, \\ 69364 Lyon Cedex 7, France
}

(Received 2 October 2015; published 30 December 2015)

\begin{abstract}
Gas invasion in liquid-saturated sands exhibits different morphologies and dynamics. For mobile beds, the repeated rise of gas through the layer leads to the growth of a fluidized zone, which reaches a stationary shape. Here, we present experimental results characterizing the evolution of the fluidized region as a function of the gas-flow rate and grain size. We introduce a new observable, the flow density, which quantifies the motion of the grains in the system. The growth of the fluidized zone is characterized by a spatiotemporal analysis, which provides the stabilization time, $\tau_{s}$. In the stationary regime, we report two main contributions to motion in the fluidized region: the central gas rise and a convective granular motion. Interestingly, a static model with a fixed porous network accounts for the final shape of the invasion zone. We propose an explanation where the initial gas invasion weakens the system and fixes since the early stage the morphology of the fluidized zone.
\end{abstract}

DOI: 10.1103/PhysRevE.92.062210

PACS number(s): 83.80.Fg, 47.57.Gc, 47.85.Dh

\section{INTRODUCTION}

Gas invasion in liquid-saturated porous media has been widely studied over the past years, and is still an active research topic, due to its numerous applications in many different fields. Phenomena like air sparging [1-3], $\mathrm{CO}_{2}$ sequestration [4], catalytic processes [5] or hydrothermal complexes [6,7], methane venting areas [8-10], and volcanic events [11-13] are among the many industrial and geophysical fields governed by such processes. A classical approach to understand and predict the dynamics of fluid invasion has been to consider a rigid porous medium, which makes it possible to directly compare analytical or numerical models to laboratory experiments of packed grains [14,15]. This approach has opened the way to a large variety of percolation models, from the pioneering work of Witten and Sander on diffusion-controlled aggregation $[16,17]$ to later cluster formation and growth models such as the Eden or Vold-Sutherland models [18-24]. The characteristic of these invasion percolation models is the absence of gravity and viscous effects, and the formation of clusters governed by the distribution of capillary overpressure in the network. Introducing the gravity-field gradient into the system leads to either stable [14,25,26] or unstable [27-29] invasion.

In spite of the extensive theoretical and numerical models, the description of the different flow patterns in buoyancydriven, rigid porous media is still an active research topic, as it has direct consequences in hazard management $[2,30$ 32]. Studies have focused in particular on the gas invasion region, for instance, in the case of a single injection point. Indeed, predicting the morphology of this region makes it possible to infer the consequences of pollutant propagation, the extension of gas pocket leakage in sediments, or the most efficient air sparging strategy. However, real porous media may be unconstrained, nonrigid networks, e.g., loose marine sediments or soils. In such systems, the hydrodynamic coupling between the gas and liquid flow on one hand, and the grain motion on the other hand, rules the dynamics of the system. Predictions of the gas invasion pattern in these systems is more complex, and may include local fluidization of the medium, as well as temporal evolution and instabilities of the gas flow [33,34]. In particular, the existence of a fluidized zone with a parabolic shape above the gas injection point has been reported in the stationary regime [1,32,33,35-37]. Interestingly, this shape is very similar to the invasion pattern found in rigid porous network, where the invasion zone can be defined as the superposition of the different possible gas pathways in the system [30,31,36,38]. Recent works pointed out this similarity and found that the morphology of the fluidized zone can be predicted by a simple analytical model, where the grain matrix is fixed. This apparent contradiction, however, has to be unraveled.

In this work, we analyze the morphology and dynamics of the fluidized zone generated by a continuous air flow at a single injection point at the bottom of a liquid-saturated granular bed (Sec. II). We develop an image analysis technique to quantify the motion in the system (Sec. III). The fluidized zone grows, until it remains stable after a typical time $\tau_{s}$ (Sec. IV A). In the stationary regime, the two main contributions to motion in the system are quantified: the central air rise and the surrounding grain motion (Sec. IV B). Both the average motion over the whole experimental time and the temporal evolution of the motion distribution in the granular bed are analyzed as a function of the system parameters, namely the gas injection flow rate and the grain size. The results are compared with numerical simulations based on the hypothesis of a rigid porous network.

We demonstrate that, contrary to the intuition, the propagation of air in a rigid, saturated porous network can account successfully for the stationary shape of the fluidized zone-although it cannot, of course, capture its dynamics. The dependence of its morphology on the parameters (grain size and flow rate) is found to be similar both in the experiment (as shown here) and in previous numerical simulations computing the air invasion in a rigid, immersed granular bed.

\section{EXPERIMENTAL SETUP}

The setup consists of a vertical Hele-Shaw cell (width $40 \mathrm{~cm}$, height $30 \mathrm{~cm}$, gap $e=2 \mathrm{~mm}$ ) filled with polydisperse 


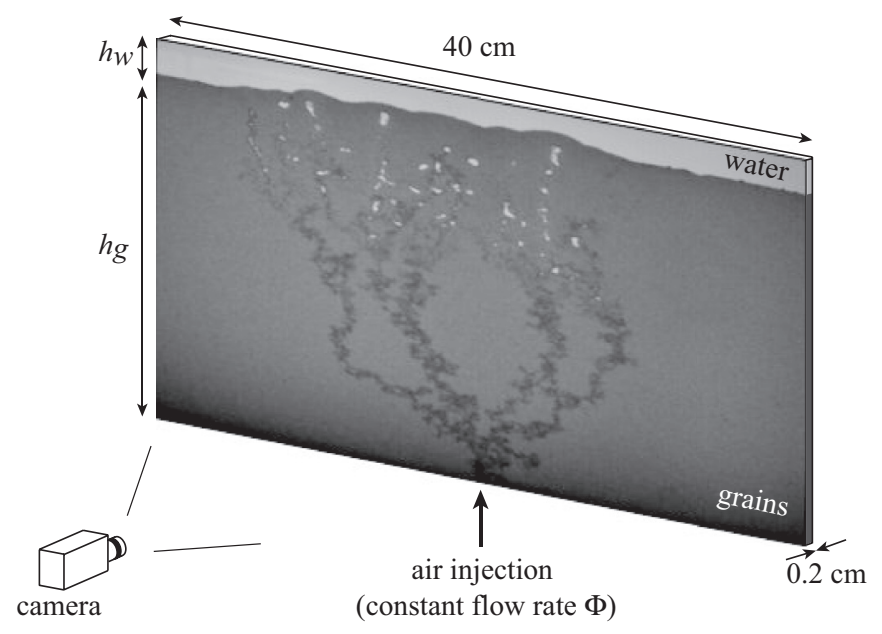

FIG. 1. Experimental setup. Air is injected at constant flow rate through an inlet (diameter $2 \mathrm{~mm}$ ) into a thin vertical cell (width $40 \mathrm{~cm}$, gap $0.2 \mathrm{~cm}$ ). Images are recorded with a camera positioned in front of the cell. The image presented is obtained for early injection when the system is mainly in the percolation regime and multiple bubbles emissions are observed at the surface.

glass beads immersed in water (Fig. 1). The gap $e$ is chosen such as only a few grains fill the gap (quasibidimensional system). Air is injected at the bottom of the cell at a single point at a constant flow rate $\Phi$ (from 0.16 to $3.3 \mathrm{~mL} / \mathrm{s}$ ), controlled by a digital mass flow controller $(\mathrm{M}+\mathrm{W}$ Instruments, model D-6311). An array of LED ensures a high and constant intensity of light over the entire cell (backlight). To ensure reproducible experiments, the initial condition is obtained as follows. The cell is filled with the beads (Sovitech glass spheres), previously sieved in order to control their size. The cell is then filled with water, up to the top boundary, and flipped bottom-up, which forces most of the small air bubbles that may still be trapped in the system to escape the granular layer and rise to the surface. While the grains are not fully deposited (very loose packing), we flip the cell back to its original vertical position. All the small bubbles initially trapped in the bulk are thus extracted from the system. We let the grains deposit, and the free surface is gently leveled with a small paddle. We denote $h_{g}$ the height of the granular layer (here, $h_{g}=20 \mathrm{~cm}$ ), and fix a water height $h_{w}=2 \mathrm{~cm}$ above the grains' free surface. This protocol ensures a reproducible granular bed (loose packing).

Four different grain batches have been used in the experiments. In Table I, we report the grains' diameter for the four batches. In order to get a precise grain-size measurement, the

TABLE I. Grains diameter from sieving and from direct measurement (average and standard deviation from Fig. 2) for the four different grains batches.

\begin{tabular}{ccc}
\hline \hline $\begin{array}{c}\text { Batch } \\
\#\end{array}$ & $\begin{array}{c}d[\mu \mathrm{m}] \\
\text { (from sieving) }\end{array}$ & $\begin{array}{c}d[\mu \mathrm{m}] \\
\text { (measured) }\end{array}$ \\
\hline 1 & $200-250$ & $218 \pm 17$ \\
2 & $250-425$ & $318 \pm 44$ \\
3 & $600-710$ & $631 \pm 37$ \\
4 & $710-965$ & $802 \pm 68$ \\
\hline \hline
\end{tabular}
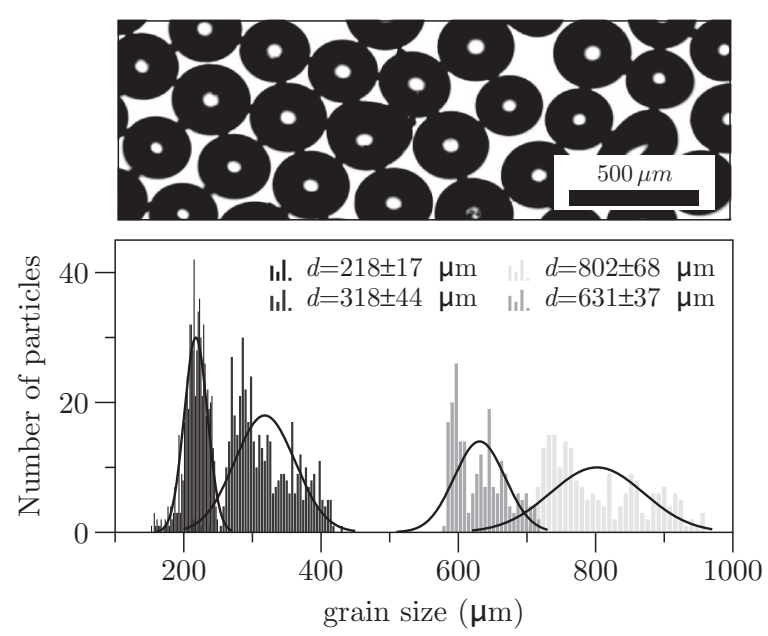

FIG. 2. Particle-size distribution: image of the glass beads and size distribution for the four different batches. Gaussian interpolations, respectively, lead to $d=218 \pm 17 \mu \mathrm{m}$ (batch \#1), $d=$ $318 \pm 44 \mu \mathrm{m}$ (batch \#2), $d=631 \pm 37 \mu \mathrm{m}$ (batch \#3), and $d=$ $802 \pm 68 \mu \mathrm{m}$ (batch \#4).

average diameter and standard deviation are determined from direct visualization and subsequent image analysis (see Fig. 2). The results are reported in Table I. In the following, we shall use the measured values to refer to the grain size.

The evolution of the system is recorded with a camera (PixeLINK, model PL-B741U, $1280 \times 800 \mathrm{px}^{2}$ ) located in front of the cell (Fig. 1), capturing one image every $10 \mathrm{~s}$ $(\Delta t=10 \mathrm{~s})$. The acquisition rate is chosen such that only a few air channels form between successive images. We thus obtain data with a sufficient temporal resolution while keeping their size reasonable.

\section{IMAGE ANALYSIS AND NUMERICAL SIMULATIONS}

\section{A. Flow-density computation}

In order to track the grains dynamics, we quantify the grain motion in the cell by analyzing the absolute difference in the intensity of two consecutive images, $\left|\mathrm{I}_{k+1}-\mathrm{I}_{k}\right|$. This matrix contains either zero or nonzero elements corresponding to regions where no motion (respectively, motion) occurred. However, the generation of the fluidized zone corresponds to a cumulative process due to the continuous gas emission. Therefore, its dynamics is generally slow and requires accumulating information over time. Hence, we define the flow density, $\rho_{n}(x, z)$, obtained by summing the consecutive image differences as follows [37]:

$$
\rho_{n}(x, z)=\sum_{k=1}^{n-1}\left|\mathrm{I}_{k+1}-\mathrm{I}_{k}\right| .
$$

The normalized flow density is then defined as $\bar{\rho}=$ $\rho_{n} / \max \left(\rho_{n}\right)$. This latter observable provides an average map of the grain motion in the cell, where the limit between the compacted zone and fluidized zone can be clearly distinguished [Fig. 3(a)]. The boundary of the fluidized zone is determined by fixing a low threshold value, here $\bar{\rho}=0.1$ [white dashed line, Fig. 3(a)]. The different regions in the granular layer can be 


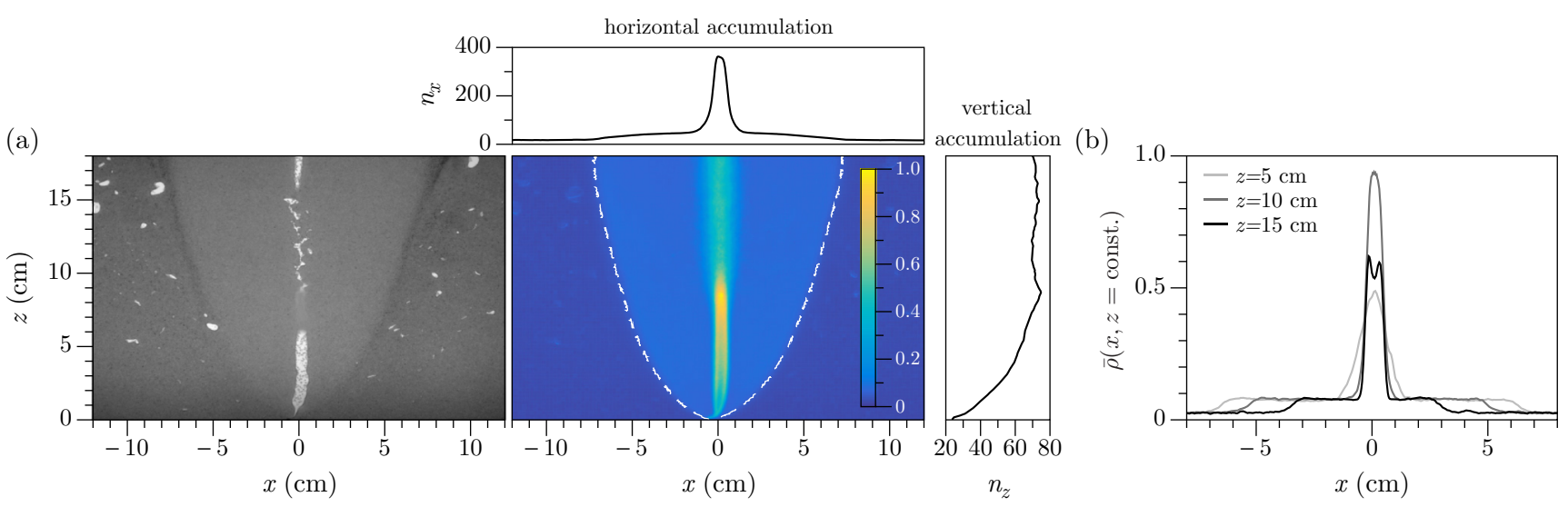

FIG. 3. (Color online) Image analysis and flow density computation: (a) Left, image of the experiment after $t \simeq 5 \mathrm{~h}[d=318 \pm 44 \mu \mathrm{m}$, $\phi=0.66 \mathrm{~mL} / \mathrm{s}]$. We observe a fluidized zone at the center (light gray) and a compacted zone on both sides (dark gray). Right, normalized flow density $\bar{\rho}$ computed through the cumulation of successive image differences, up to the same time $t \simeq 5 \mathrm{~h}$. The higher values [yellow (light color)] correspond to zones where more motion occurred (typically located at the center of the cell), whereas the lower values [blue (dark color)] correspond to zones with less or no motion. The white dashed line indicates the boundary between the fluidized zone and the compacted zone, defined as $\bar{\rho}=0.1$. The top (respectively, right side) panel represents the horizontal (respectively, vertical) cumulation value of the pixels for each row, $n_{x}$ (respectively, $\left.n_{z}\right)$. (b) Examples of $\bar{\rho}(x, z=c t e)$ profiles for three different heights. Note the plateau inside the fluidized zone, indicating a homogeneous motion, and a large central peak corresponding to the air channel contribution.

observed by representing different profiles of the normalized flow density, $\bar{\rho}$, at a constant height [Fig. 3(b)]. The boundary of the fluidized zone is clearly defined for all heights. We report two main contributions to the flow density in the fluidized region: a central peak, corresponding to the central air channel, and a plateau ( $\bar{\rho}$ constant), indicating a homogeneous motion.

To further quantify the grain motion, we define the horizontal $\left(n_{x}\right)$ and vertical $\left(n_{z}\right)$ cumulations as the sum of pixels for each row $\left(n_{x}\right)$ and column $\left(n_{z}\right)$ in the density profile:

$$
n_{x}(x, t)=\sum_{z} \rho_{n}(x, z) ; \quad n_{z}(z, t)=\sum_{x} \rho_{n}(x, z)
$$

where $t$ is the time corresponding to the $n$th image, $t=n \Delta t$, and their normalized value,

$$
\begin{aligned}
\bar{n}_{x}(x, t) & =n_{x}(x, t) / \max \left[n_{x}(x, t)\right], \\
\bar{n}_{z}(z, t) & =n_{z}(z, t) / \max \left[n_{z}(z, t)\right] .
\end{aligned}
$$

These quantities provide extra information and make it possible to quantify not only the geometrical aspects of the fluidized zone [Fig. 3(a)] but also its temporal evolution (Sec. IV A).

\section{B. Numerical simulations}

Previous studies pointed out that the global morphology of the zone invaded by the gas can be accounted for by a simple model considering a fixed porous network $[36,39,40]$. However, why the air path through a rigid granular matrix apparently captures the geometry of a convective, fluidized zone is still under debate. To further investigate this issue, the experimental results presented in this work are compared with numerical simulations, including the same basic ingredients, which are summarized as follows. The granular packing can be reduced, in a simple manner, to a $2 \mathrm{D}$ square network where each vertex corresponds to the void space between the four neighboring grains. Each vertex is associated with a random value of the capillary overpressure obtained from a Gaussian distribution accounting for the heterogeneity of the system (grains polydispersity and local packing fraction). A vertical pressure gradient representing the hydrostatic pressure is added. To propagate through the network, the gas has to overcome the weakest capillary overpressure associated with all the vertices along its path (see Ref. [39] for more details). The size of the network is changed depending on the grain size and is limited to match the total size of the experimental cell. In contrast to previous works [39,40] and to compare with the experiments, we do not consider here a fixed volume of gas injected in the network, but let the air propagate until reaching the surface.

As previously reported [39], the system dynamics is governed by a single dimensionless parameter that compares the relative effects of gravity and capillarity and takes into account the heterogeneity of the grain network, $\chi=\sigma_{p} / \rho g d$, where $\rho$ is the water density, $g$ the gravitational acceleration, $d$ the typical grain diameter, and $\sigma_{p}$ the width of the distribution of the capillary overpressures in the system. By considering $w$, the typical relative variation of the pore size, and capillary overpressure of the order of $\gamma / d$ where $\gamma$ is the air-water surface tension, we can write

$$
\chi=\frac{w \gamma}{\rho g d^{2}} .
$$

For simplicity, unless specified, $w \simeq 1$. For each value of the parameter $\chi$, the density map of gas invasion in the granular layer is obtained by adding 3000 runs.

\section{Preliminary results}

To compare the numerical simulations with experimental results, we consider similar grain diameter, $d=631 \pm 37 \mu \mathrm{m}$ (batch \#3), corresponding to $\chi \simeq 15$, and plot the normalized flow density $\bar{\rho}$ [Figs. 4(a) and 4(b)]. In the numerical 
(a)

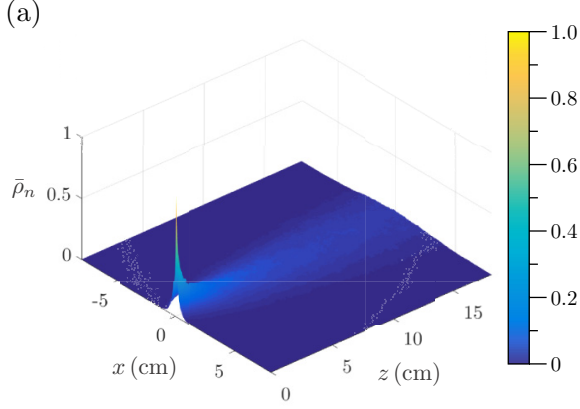

(b)

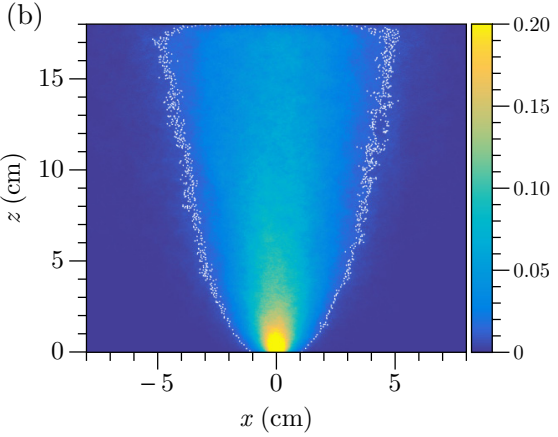

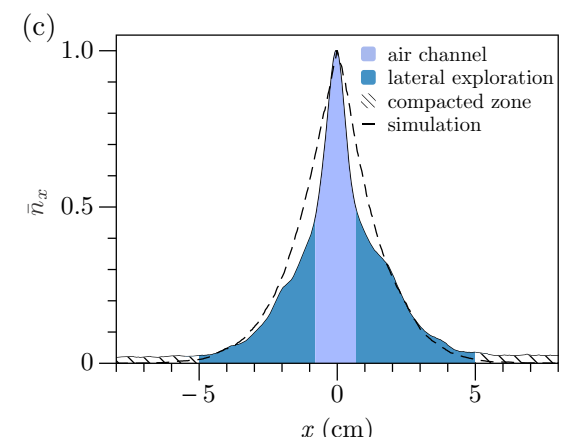

FIG. 4. (Color online) Numerical simulation: (a) Surface plot for the normalized flow density $\bar{\rho}$ obtained numerically for conditions similar to the experiment ( $\chi \simeq 15$ corresponding to the grain batch $\# 3, d=631 \pm 37 \mu \mathrm{m}$ ). The results point out a maximum concentration at the injection point. (b) Alternative view for the normalized flow density where the scale is limited to $\bar{\rho}_{\max }=0.2$ to visualize the outmost contour. The white points represent the boundary for the fluidized zone (threshold $\bar{\rho}=1 \%$ ). (c) Normalized horizontal cumulation $\bar{n}_{x}$ comparison for the experiment (continuous line) and simulation (dotted line). The experimental case displays a strong contribution at the center, due to the central air channel (see text).

simulation, it is interesting to note the highly concentrated flow density at the injection point, which then decays rapidly to cover the entire area [Fig. 4(a)]. We define the invasion zone as the region explored by the gas and qualitatively compare this zone to the fluidized zone observed in the experiments, as done in previous work [36]. We consider a threshold of the order of a few percent for the flow density [39], here $\bar{\rho}=1 \%$, and plot the boundary [white dots, Fig. 4(b)]. Similar to the experiment and to previous results [36,39], the central invasion region displays a parabolic profile.

It is particularly interesting to report the normalized horizontal cumulation, $\bar{n}_{x}$, for both the numerical simulation and experimental cases [Fig. 4(c)]. The results display similar profiles sharing roughly the same width at the base (corresponding to the average width of the invasion zone or fluidized zone), but exhibit some differences in the upper part of the profile. Indeed, in the numerical simulation, the profile exhibits a smooth evolution from the center (injection point) to the borders, indicating that the movement is evenly distributed throughout the cell [dotted line, Fig. 4(c)]. In contrast, the experimental results can be separated in three regions [Fig. 4(c)]: (i) a central peak (light blue), (ii) a wider distribution around the center (dark blue), and (iii) a plateau on both sides (hatched zones). The central peak corresponds to the air channel, which forms when air pushes the grains during its rise through the cell. This major contribution is stable in time and gives the higher amplitude in the horizontal accumulation. It can be simply explained by noting that each air path through the granular layer creates a weaker path for the following bubbles, leading to a central channel stabilization. The second region corresponds to the intermittent branches (air percolation or fractures), which accumulation gives a lateral contribution in a lesser extent. The last region on both sides (plateau in $\bar{n}_{x}$ ) corresponds to a static zone, located out of the border of the invasion zone, which air does not explore.

\section{Limitations}

The results provided by the numerical simulation cannot be extended to all the grain sizes and flow rates. For smaller grain sizes, in particular (mainly for the batch \#2, $d=318 \pm$ $44 \mu \mathrm{m})$, an additional effect is observed in the experiment.
The grains follow a global convective motion, climbing up near the center while entrained by the central air channel, and descending along the borders of the fluidized zone. This convective motion was already described in Ref. [40], and although the final parabolic shape of the invasion (numerical) and fluidized (experimental) regions can be accurately described by a parabolic profile, the global convection cannot be accounted for by the numerical simulation, which considers a static grains network. In this latter, the flow density is only produced by the accumulation of independent paths that mimic air percolation through the network. This invasion process is close to what is observed for the larger grains but does not correspond to the typical case for all sizes (specially small grains size). Note that although the motion inside the invasion zone or fluidized zone is very different between simulation and experiment, it is puzzling, however, to find a good qualitative agreement with the final parabolic shape marking the invasion zone or fluidized zone boundary. This point is discussed in detail in Sec. V.

To describe the grain motion inside the fluidized region more precisely, we investigate experimentally its dependence on flow rate and grain size, estimate the time necessary to reach a stationary state, and quantify the respective influence of the air channel and the lateral motion.

\section{DYNAMICS OF THE FLUIDIZED ZONE}

In this section, we study the dynamics of the fluidized zone generated by the continuous rise of air through the granular layer. In particular, we shall focus on the subsequent grains motion. In a first part we describe the formation of the fluidized zone at short times (Sec. IV A) and quantify the stabilization time of the central air channel as a function of the flow rate $\Phi$ and grain size $d$. We then analyze the horizontal cumulation $\bar{n}_{x}$ for all data, in the stationary state, and discuss a tentative model to interpret the results in terms of grain motion inside the fluidized zone (Sec. IV B).

\section{A. Transient: Growth of the fluidized zone}

At the beginning of the gas invasion, air propagates through the granular matrix, producing small local rearrangements. 

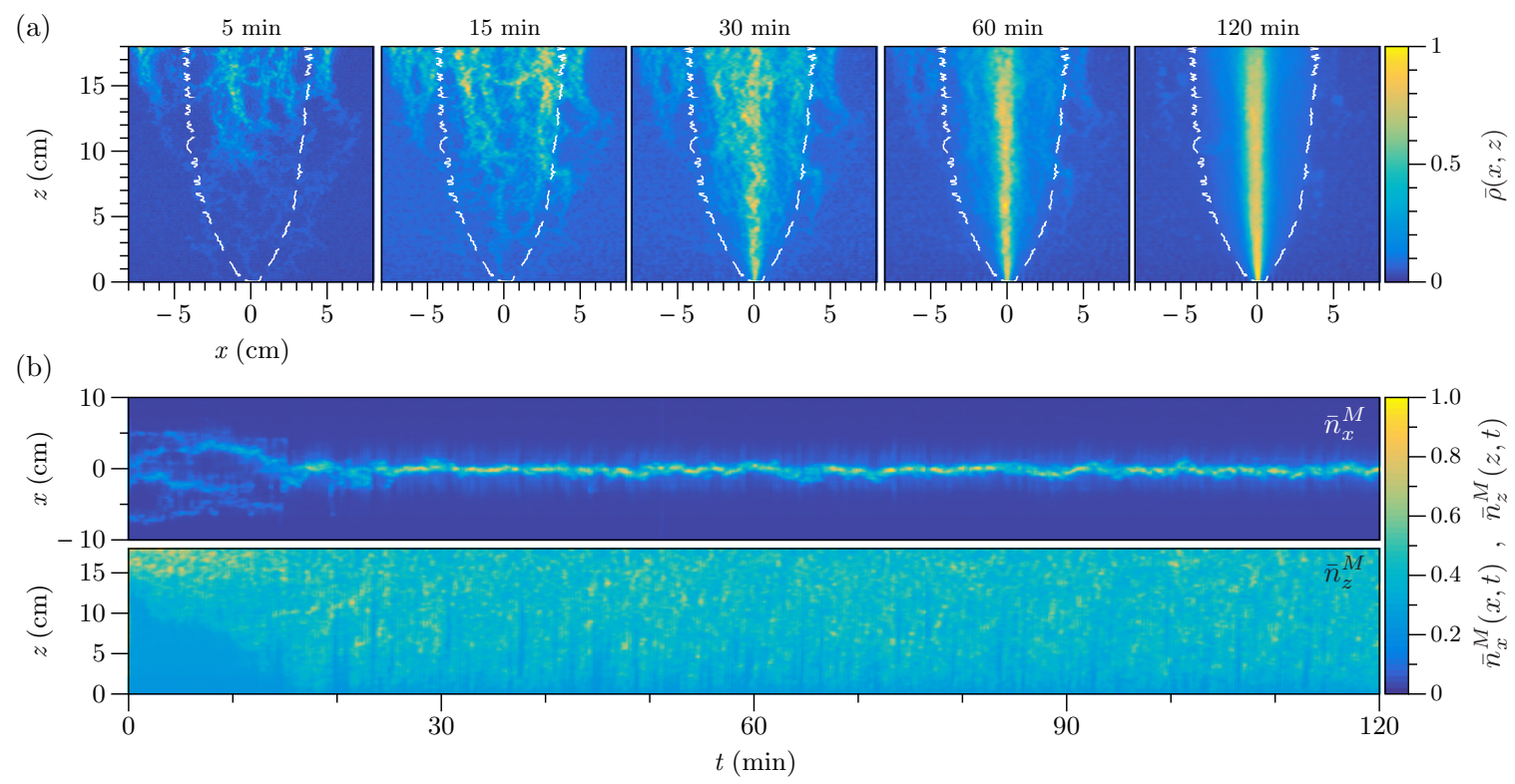

FIG. 5. (Color online) Evolution of the fluidized zone: (a) Normalized flow density $\bar{\rho}$ at different time after starting the gas invasion through the system. The images show the grain motion and the later formation of the fluidized zone at different stages. At the beginning, the motion focuses mainly close to the grains' free surface, then propagates downward in time. After a few minutes $(\sim 20$ min) a central air channel is formed, and the air paths explore less and less the surroundings. Note that the formation of air branches at the first stage of the invasion covers a wider area than the final geometry of the fluidized zone. The dashed lines correspond to the border of the fluidized zone defined at long times $[\Phi=0.66 \mathrm{~mL} / \mathrm{s}, d=318 \pm 44 \mu \mathrm{m}]$. (b) Spatiotemporal diagram of the two quantities $\bar{n}_{x}^{M}(x, t)$ (up) and $\bar{n}_{z}^{M}(z, t)$ (down) for the same parameters. $\bar{n}_{x}^{M}$ characterizes the formation of the central air channel and the fluidized zone extent, while $\bar{n}_{z}^{M}$ helps describe the distribution of motion along the cell vertical axis. The stabilization time $\tau_{s}(\sim 18 \mathrm{~min}$, dotted line) corresponds to the first value found for a threshold of $\bar{n}_{x}^{M}(x, t)=0.7$ (see text).

After reaching the free surface, it starts being emitted as small bubbles through the topmost part of the granular bed, which concentrates most of the motion [Fig. 5(a), left panel]. Note that at this stage, the gas explores an area wider than the final geometry of the fluidized zone [white dotted line, Fig. 5(a)]. The gas emissions then stabilize, and in time, the region affected by the grain motion propagates downward, towards the injection nozzle [Fig. 5(a)]. The motion then focuses along the vertical axis above the gas inlet, with the intermittent emergence of lateral branches. This last behavior is stable at long times and marks the onset of the formation of the fluidized zone. In this region, the grains exhibit a convective motion, climbing up along the central axis while entrained by the rising gas and descending along the sides, thus forming two symmetric convection rolls [40].

To quantify the growth and stabilization of the fluidized zone, we consider the moving average of the flow density in time, computed over $M$ images:

$$
\rho_{n}^{M}(x, z)=\sum_{k=n-M}^{n-1}\left|\mathrm{I}_{k+1}-\mathrm{I}_{k}\right| .
$$

In the following, we consider $M=10$. In a similar way, we define the moving average of the horizontal and vertical cumulation as

$$
\begin{aligned}
& n_{x}^{M}(x, t)=\sum_{z} \rho_{n}^{M}(x, z), \\
& n_{z}^{M}(z, t)=\sum_{x} \rho_{n}^{M}(x, z) .
\end{aligned}
$$

We normalize these two quantities by their maximum value obtained over the whole experimental time, and get, respectively, $\bar{n}_{x}^{M}(x, t)$ and $\bar{n}_{z}^{M}(z, t)$. The former is an accurate parameter to characterize the formation of the central air channel and the fluidized zone extent, while the latter helps describing the distribution of motion along the cell vertical axis. Figure 5(b) displays the spatiotemporal diagrams of these two quantities. At the early stage of gas invasion, we clearly see a wide exploration of the gas pathways along the horizontal axis [Fig. 5(b), top panel], while most of the motion is concentrated in the topmost part of the granular bed [Fig. 5(b), bottom panel]. The evolution of $\bar{n}_{z}^{M}$ in time shows the downward propagation of motion, until the system stabilizes. At this stage, the final shape of the fluidized zone is well-defined and does not grow anymore. Note that once the system reaches this stationary state, almost all the motion is concentrated along the central, vertical axis [Fig. 5(b), top panel] and is distributed roughly homogeneously in $z$ [Fig. 5(b), bottom panel], indicating the stabilization of a central air channel.

The time required to stabilize the air channel, $\tau_{s}$, can be very different depending on the experimental parameters. Figure 6(a) displays two spatiotemporal diagrams of the normalized horizontal cumulation $\bar{n}_{x}^{M}(x, t)$ for two different flow rates $(\Phi=0.16$ and $2.0 \mathrm{~mL} / \mathrm{s})$ and same grain size $[d=218 \pm 17 \mu \mathrm{m}]$. For small flow rate [Fig. 6(a), top panel] we observe that several air channels explore different positions, they are created and destroyed until a single one achieves stabilization at the center of the cell. For larger flow rate [Fig. 6(a), bottom panel], the air is rapidly focused on a single centered channel and remains stable in time. In both cases, the 

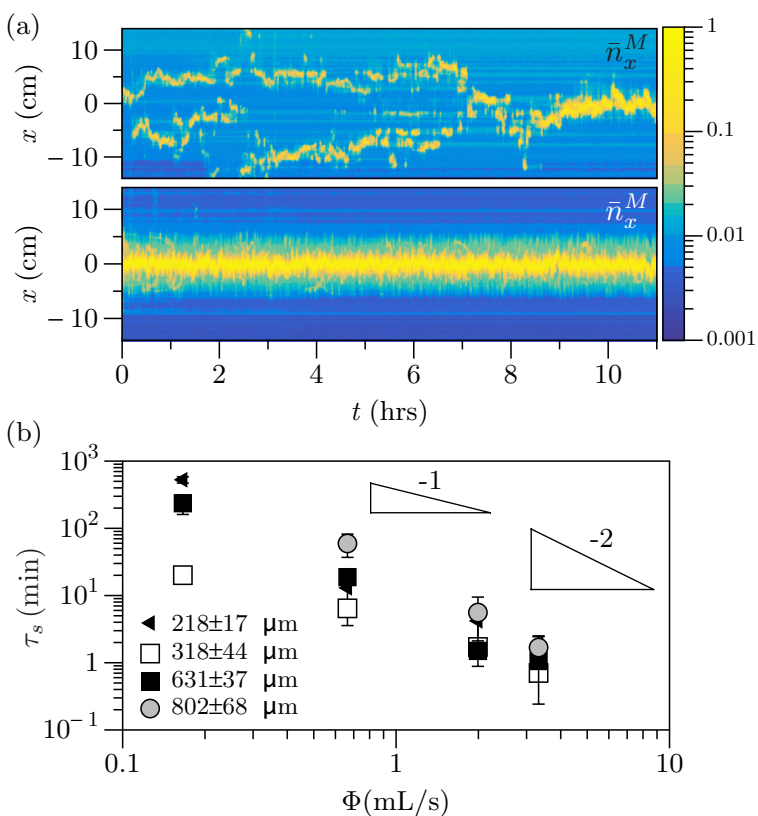

FIG. 6. (Color online) Stabilization of the air channel: (a) Spatiotemporal diagram of $\bar{n}_{x}^{M}(x, t)$ for the same grain size $[d=218 \pm$ $17 \mu \mathrm{m}]$ and different injection flow rates $[\Phi=0.16 \mathrm{~mL} / \mathrm{s}$ (up) and $\Phi=2.0 \mathrm{~mL} / \mathrm{s}$ (down)]. The stabilization of the air channel depends roughly on the air flow rate $\left(\tau_{s} \sim 9 \mathrm{~h}\right.$ for the low flow rate and a few minutes for the higher flow rate). The normalized cumulations (color bar) are presented in logarithmic scale to highlight small values. (b) Characteristic stabilization time $\tau_{s}$ as a function of the flow rate, for different grain batches. The triangles indicate the slopes -1 and -2 in $\log -\log$ plot.

importance of the gas propagation history has to be underlined. Indeed, when a channel is formed locally, it generates a lowdensity area, which acts as a weaker barrier and a preferential path for the gas, and promotes its stabilization. In addition, the effect of inertia, more important for higher flow rates, tends to favor vertical motion and reduce lateral explorations.

To set a unique criterion and quantify the characteristic stabilization time, we define $\tau_{s}$ as the first time when the normalized horizontal cumulation takes a threshold value of $\bar{n}_{x}^{M}\left(x, \tau_{s}\right)=0.7$. This threshold value is chosen small enough to capture correctly the stabilization time and large enough to prevent further formation of small intermittent channels of lateral gas exploration [Fig. 5(b)]. Note that no fluidized zone, and hence no stabilization time, can be defined for $\Phi=$ $0.16 \mathrm{~mL} / \mathrm{s}$ and the grain batch \#4 $(d=802 \pm 68 \mu \mathrm{m})$. This experiment, corresponding to the smaller flow rate and larger grain size explored in the experiments, will not be considered in the following. Figure 6(b) displays the stabilization time $\tau_{s}$ for all the grains size and flow rate. Although a previous work reported a stabilization time $\tau_{s} \sim 1 / \Phi$ for fluidization of a two-phase system [41], the results reported here point out a decrease of $\tau_{s}$ with increasing $\Phi$ as $\tau_{s} \sim 1 / \Phi^{2}$, independently of the grain size. Further interpretation of this result is provided in the discussion (Sec. V).

\section{B. Stationary state: Motion in the fluidized zone}

To characterize the dynamics in the fluidized zone in the stationary regime, i.e., after it is fully developed, we compute the normalized horizontal cumulation $\bar{n}_{x}$ as a function of $x$ for the different grain size and gas flow rates [Fig. 7]. The results presented here are for the same total experimental time ( $t_{\mathrm{tot}}=12 \mathrm{~h}$ after starting gas injection). For all experiments, the system after this time is in a well-established, stationary regime, where the air channel has reached its steady-state central location $\left(t_{\text {tot }}>\tau_{s}\right)$. For small grain size $(d=218 \pm$ $17 \mu \mathrm{m}), \bar{n}_{x}$ displays a single, central peak, indicating that the motion mainly concentrated along the vertical axis, at the center of the cell. The gas rises as a central channel, and the grain motion is located in its close neighborhood,
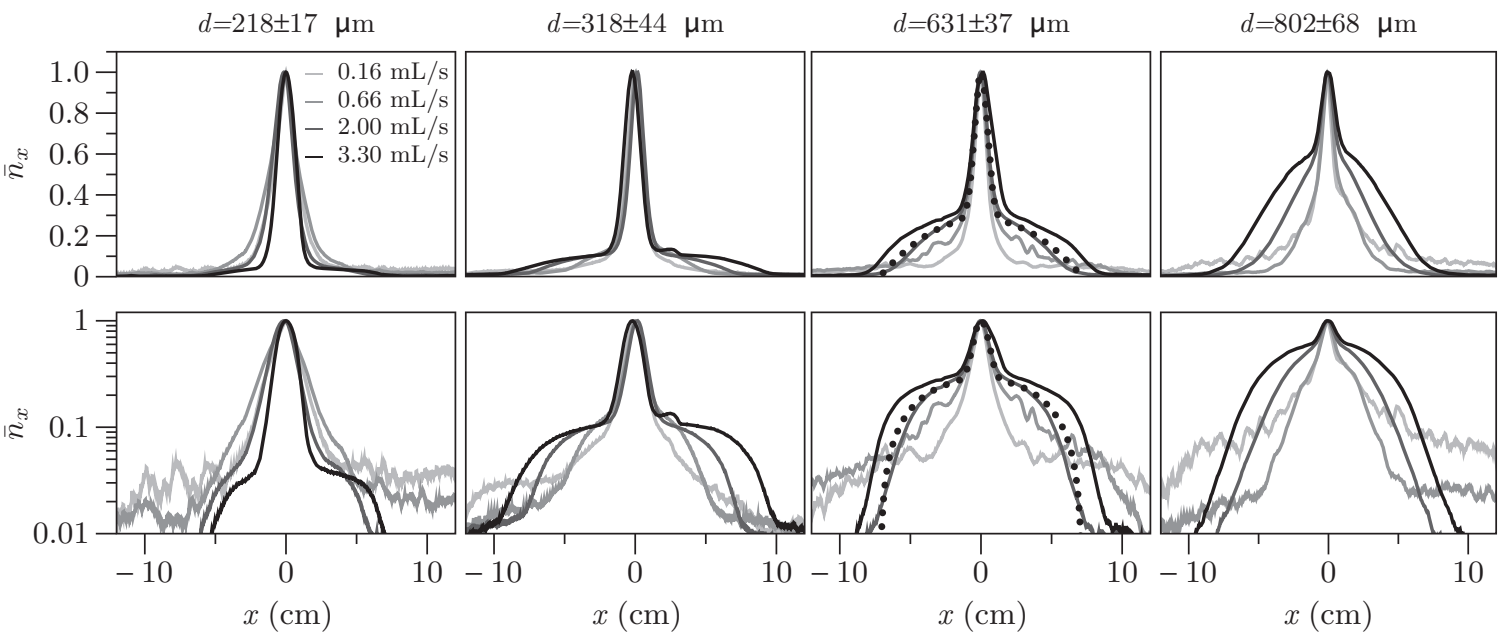

FIG. 7. Normalized horizontal cumulation $\bar{n}_{x}$ for different grain size $d$ and gas flow rate $\Phi$ (lin-scale, top panel; log-scale, bottom panel). The experimental result shows that the profiles for a small grain size and different flow rates share a similar geometry characterized by a single central peak. When increasing the grain size, the profile deforms and its base develops a larger distribution. This contribution comes mainly from the convective motion of grains in the fluidized zone $(d<425 \mu \mathrm{m})$ or from the formation of sides branches (typically viscous fingering and fracturing) that spread the motion along the horizontal axis $(d>600 \mu \mathrm{m})$. The black dots correspond to the results of the fit using Eq. (11) for the data corresponding to $d=631 \pm 37 \mu \mathrm{m}$ and $\Phi=2.0 \mathrm{~mL} / \mathrm{s}$. 

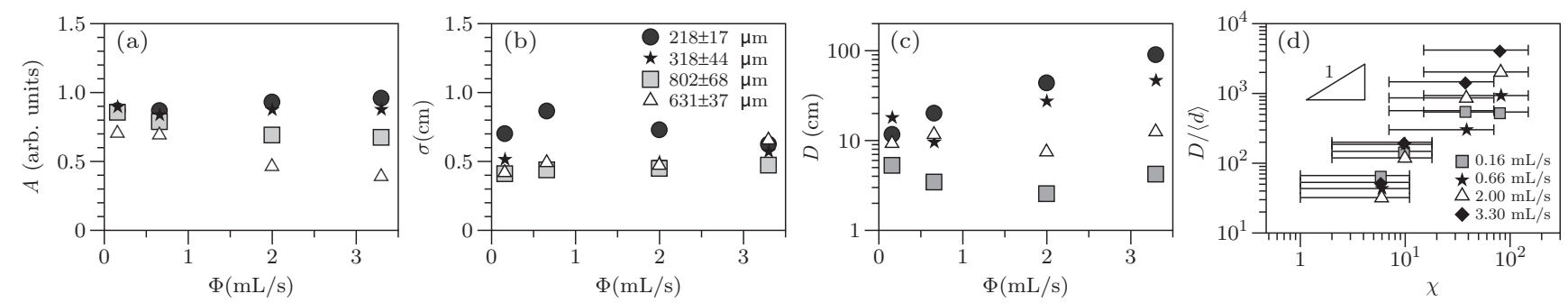

FIG. 8. Parameters describing the two components of motion in the fluidized zone (see text). (a), (b) Amplitude, $A$, and standard deviation, $\sigma$, of the Gaussian function characterizing the central air channel, as a function of the flow rate $\Phi$, for different grain size $d$. (c) Coefficient $D$ characterizing the parabolic shape of the fluidized zone, $z=x^{2} / D$, as a function of the flow rate $\Phi$, for different grain size [same legend as (b)]. (d) Coefficient $D$ normalized by the average grain size for each batch, as a function of the parameter $\chi$ (see text). The triangle indicates the slope 1 .

while no motion is observed on both sides. When increasing the grain size, we still observe the central peak due to the air-channel contribution, but a lower and wider area becomes more and more predominant. For intermediate grain diameter ( $d=318 \pm 44 \mu \mathrm{m})$, direct visualization makes it possible to correlate this second contribution to the grains convection in the fluidized zone. For large grain size $(d=631 \pm 37$ and $802 \pm 68 \mu \mathrm{m}$ ), the exploration of gas becomes more complex. In this case, the wider base, superimposed on the central peak, is not produced by the convection of grains, but by air paths that remain stable over long times. Interestingly, as already reported in previous work, the final geometry of the region where most of the motion occurs has the same parabolic shape, whichever the gas invasion mechanism (percolation, fracture, or channel inducing grain convection in the fluidized zone) $[36,40]$.

To further quantify the contribution of the different mechanisms to the global motion in the system, we propose to separate the central, strong motion to the mean grain flow in the fluidized zone. The main hypothesis is that the mean grain flow in the fluidized zone is spatially homogeneous, of amplitude $c$. This flow is limited by the borders of parabolic shape, $z=x^{2} / D$, where $D$ may depend on the experimental parameters (gas flow rate or grain size). We then add the contribution of the central air channel, taken as a Gaussian of amplitude $a$ and standard deviation $\sigma$, centered at $x=0$, $z=a \exp \left(-x^{2} / 2 \sigma^{2}\right)$. No motion is considered outside the fluidized zone. The horizontal cumulation can thus be written

$$
n_{x}(x)=\int_{\frac{x^{2}}{D}}^{h_{g}} c d z+\int_{0}^{h_{g}} a \mathrm{e}^{-\frac{x^{2}}{2 \sigma^{2}}} d z .
$$

Integrating these simple expressions and normalizing leads to

$$
\bar{n}_{x}(x)=\left(\frac{c}{c+a}\right)-\frac{c}{h_{g}(c+a)} \frac{x^{2}}{D}+\left(\frac{a}{c+a}\right) e^{-\frac{x^{2}}{2 \sigma^{2}}} .
$$

Rewriting $A=a /(c+a)$ and $D^{\prime}=D h_{g}(c+a) / c$ gives

$$
\bar{n}_{x}(x)=(1-A)-\frac{x^{2}}{D^{\prime}}+A e^{-\frac{x^{2}}{2 \sigma^{2}}} .
$$

Fitting the experimental data with this function gives an excellent agreement (black dots in Fig. 7). We determined the parameters $\left(A, \sigma, D^{\prime}\right)$ for all the experimental data.

First, let us focus on the parameters (amplitude and standard deviation) characterizing the central air rise, where the strongest motion locates. Neither the amplitude $A$ nor the standard deviation $\sigma$ depend significantly on the flow rate [Figs. 8(a) and 8(b)], meaning that the contribution of the central air rise is roughly the same from all experiments. Note the typical value of the standard deviation, $\sigma \sim 0.5 \mathrm{~cm}$, consistent with the observations of a central air channel width of about $1 \mathrm{~cm}$. The fact that the expression given in Eq. (11) fits well the experimental data validates the hypothesis of a homogeneous motion in the fluidized zone, superimposed with a central air rise. The coefficient $D$, which characterizes the parabolic shape of the fluidized region, is of the same order of magnitude $(\sim 10 \mathrm{~cm})$ as reported in a previous work [37] [Fig. 8(c)]. Note that the analysis method presented here does not need any arbitrary threshold, contrary to the procedure detailed in Refs. [37,40], but directly provides the estimation of this coefficient without any assumption. For experiments where a convective region is well-defined (small or intermediate grains), $D$ is found to increase with the flow rate [Fig. 8(c)], while for larger grains $(d>600 \mu \mathrm{m}), D$ does not exhibit any significant dependence on $\Phi$.

\section{DISCUSSION}

To discuss the experimental results in regard to the parabolic shape determined through the numerical simulations (Sec. III B), we plot $D$, normalized by the average grain size $\langle d\rangle$ for each batch, as a function of the dimensionless parameter $\chi$ given by Eq. (5) [Fig. 8(d)]. The typical relative variation of the pore size, however, is difficult to estimate. It is usually of the order of the relative width of the grain-size distribution [36], but may strongly vary from one batch to the other. To account for this dispersion, we plot the error bars associated with a typical variation of $w$ between 0.1 and 1 . The data points are plotted for the average value of $\chi$ for each batch. We find $D /\langle d\rangle \propto \chi$, similar to the relationship obtained with numerical simulations considering a rigid, porous granular matrix [39].

It is interesting to note the similarity of results for the final shape of the fluidized zone (in the experimental case) or invasion zone (for numerical simulations). The fluidized and invasion zone morphology in the stationary state does not seem to depend on its dynamics. At the early stage of gas invasion, however, the air pathways through the granular bed may be quite similar between the experiment and the numerical simulation. Indeed, the gas explores a wide part of the system without significantly moving the grains (percolation). This 
first stage may weaken the system, and the accumulation of this process during the early gas invasion defines a zone that may have the same characteristics in both the experiments and the numerical simulations.

With such a hypothesis, one could be tempted to explain the dependence of $\tau_{s}$, the characteristic stabilization time, on $\Phi$ with the simple analytical model that was first developed in the case of the rigid porous network [36]. Indeed, when the stationary regime is reached, we can estimate the total volume of air that passed through the grains as $V=\Phi \tau_{s}$. If we consider that the stationary state is obtained when, in average, the gas pathways have explored the whole invasion zone, we can write, on the other hand, $V=2 e \int x d z$, where $e$ is the gap of the cell and $x=\sqrt{D z}$ defined the border of the fluidized zone. Assuming that the final geometry is independent of the air flow rate $\Phi$ and depends only on the grains size $d$, the coefficient $D$ can be written

$$
D=d\left(\frac{1-p_{z}}{p_{z}}\right),
$$

where $p_{z}$ is the probability that the air path goes up at a given vertex in the granular matrix (see Refs. [36,39] for more information). In a first approximation, $D$ can be considered of the order of the grain size $d, D \sim d$. Thereby, we can write the total volume of air that crossed the system as

$$
V=\Phi \tau_{s}=2 e \sqrt{d} \int_{0}^{h_{g}} \sqrt{z} d z,
$$

which leads to

$$
\tau_{s} \propto \frac{e \sqrt{d} h_{g}^{3 / 2}}{\Phi} .
$$

This simple reasoning, based on the analytical model associated with a fixed porous matrix, predicts a dependence of $\tau_{s}$ on $1 / \Phi$. Although we do not expect a perfect agreement with the experiments [Fig. 6(b)], as the coefficient $D$ may depend not only on the grain size, but also the local heterogeneities (local packing fraction) or gas flow rate, the experimental results exhibit a dependence $\tau_{s} \propto 1 / \Phi^{2}$ [Fig. 6(b)], which points out another mechanism. In this case, the static assumption does not hold and dynamics has to be considered.

To account for the system dynamics, we propose a simple argument stating that the work $\mathcal{W}_{g}$ required to move a mass of grains $M_{g}$ over the total height $h_{g}, \mathcal{W}_{g}=M_{g} g h_{g}$, equals a fraction $\beta$ of the energy (work) injected in the system, $W_{i}$. This latter can be written as $\mathcal{W}_{i}=\mathcal{P}_{i} \tau_{s}$, where the injected power $\mathcal{P}_{i}=\Phi \Delta P$, where $\Delta P$ is the pressure difference. The energy balance is thus

$$
\beta \Phi \Delta P \tau_{s}=M_{g} g h_{g},
$$

where $\beta<1$. On the other hand, under laminar flow (HeleShaw cell), the pressure gradient is proportional to the flow rate, $\Delta P / h_{g}=\alpha \Phi$. It thus leads to

$$
\tau_{s}=\left(\frac{M_{g} g}{\alpha \beta}\right) \frac{1}{\Phi^{2}} .
$$

This simple energy balance, taking into account the grains dynamics, leads to a dependence $\tau_{s} \propto 1 / \Phi^{2}$, in agreement with the experimental results [Fig. 6(b)].
The expression of $\alpha$ can be derived by considering that the Poiseuille flow in the central air channel drives the motion. If considering that the channel has a constant width $w$ over the whole height, which is verified experimentally $[\sigma$ constant, Fig. 8(b)], we have $\alpha=12 \eta_{\text {air }} /\left(w e^{3}\right)$, where $\eta_{\text {air }}=1.8 \times$ $10^{-5} \mathrm{~Pa}$ is the air dynamic viscosity, $w \simeq 1 \mathrm{~cm}$ is the channel width and $e=2 \mathrm{~mm}$ the cell gap. In a crude approximation, $M_{g} \sim \rho_{g} e h_{g}^{2}$, where $\rho_{g} \simeq 2300 \mathrm{~kg} / \mathrm{m}^{3}$ is the glass beads density. We thus get $\beta \simeq\left(\rho_{g} g h_{g}^{2} w e^{4}\right) /\left(12 \eta_{\text {air }} \tau_{s} \Phi^{2}\right) \sim 10^{-3}$. From this rough estimate, we can infer that about one thousandth of the injected energy is used to move the grains in the fluidized zone.

\section{CONCLUSION}

The results reported here aim at characterizing the global dynamics produced by a continuous gas injection at the bottom of a water-saturated sand, confined in a Hele-Shaw cell. We describe the dynamic processes inside the fluidized zone formed by a gas flow forced through an immersed granular layer. The image analysis method proposed in Sec. III, coupled to a simple model superimposing a homogeneous motion in the fluidized region with a central air channel, makes it possible to quantify its final morphology, without any arbitrary threshold. Interestingly, the final shape of the fluidized zone can be captured by a simple model where the grain network is static. We interpret this result as follows. In the early stage of gas invasion in the granular matrix, the air does not significantly move the grains. The area it explores becomes more fragile, as the local packing is loosened by the gas. This specific area is roughly analog to the one explored by the gas in a rigid network. In the later stage of gas invasion, the air locates on a central channel, and for small or intermediate grain size, a convective, homogeneous motion of the grains spreads over the whole fluidized zone. We propose that this motion, forced by the central channel rise, is limited to the region that became more fragile in the early stage-hence providing a possible description of its contour with a static model.

We introduced the flow density, which gives an average information on how the motion is distributed over the granular bed and helps to characterize its dynamics. Based on this quantity, we computed the spatiotemporal evolution of the system and quantified its stabilization time $\tau_{s}$, corresponding to the time when the gas pathways focus on a central air channel, and the surrounding region, inside the fluidized zone border, moves roughly homogeneously. The dependence of $\tau_{s}$ on the gas injection flow rate $\Phi, \tau_{s} \propto 1 / \Phi^{2}$, points out that the transient involves dynamical processes and cannot be captured by the rigid network model. The local, precise characterization of the grains dynamics requires other experimental techniques (i.e., Particle Image Velocimetry), and shall be the goal of a future work.

\section{ACKNOWLEDGMENTS}

G.V. acknowledges financial support from FONDECYT Project No. 11121300. This work was supported by Programa de cooperación científica ECOS/CONICYT C14E07. 
[1] K. R. Reddy, S. Kosgi, and J. Zhou, Hazard. Waste Hazard. Mater. 12, 97 (1995).

[2] M. C. Brooks, W. R. Wise, and M. D. Annable, Ground Water Monit. Rem. 19, 105 (1999).

[3] K. R. Reddy and J. A. Adams, J. Geotech. Geoenviron. Eng. 3, 234 (2001).

[4] Q. Kang, I. N. Tsimpanogiannis, D. Zhang, and P. C. Lichtner, Fuel Process. Technol. 86, 1647 (2005).

[5] T. Zhao, T. Eda, S. Achyut, J. Haruta, M. Nishio, and M. Takei, Chem. Eng. Sci. 130, 8 (2015).

[6] H. Svensen, S. Planke, B. Jamtveit, and T. Pedersen, Geo-Mar. Lett. 23, 351 (2003).

[7] H. Svensen, B. Jamtveit, S. Planke, and L. Chevallier, J. Geol. Soc. 163, 671 (2006).

[8] H. Svensen, S. Planke, A. M.-S. Renssen, B. Jamtveit, R. Myklebust, T. R. Eidem, and S. S. Rey, Nature 429, 542 (2004).

[9] L. Naudts, J. Greinert, Y. Artemov, S. E. Beaubien, C. Borowski, and M. De Batist, Mar. Geol. 251, 253 (2008).

[10] K. R. Newman, M.-H. Cormier, J. K. Weissel, N. W. Driscoll, M. Kastner, E. A. Solomon, G. Robertson, J. C. Hill, H. Singh, R. Camilli, and R. Eustice, Earth Planet. Sci. Lett. 267, 341 (2008).

[11] S. Planke, H. Svensen, M. Hovland, D. A. Banks, and B. Jamtveit, Geo-Mar. Lett. 23, 258 (2003).

[12] V. Mastalerz, G. J. de Lange, A. Dählmann, and T. Feseker, Chem. Geol. 246, 87 (2007).

[13] A. Mazzini, H. Svensen, G. G. Akhmanov, G. Aloisi, S. Planke, A. Malthe-Sørenssen, and B. Istadi, Earth Planet. Sci. Lett. 261, 375 (2007).

[14] A. Birovljev, L. Furuberg, J. Feder, T. Jøssang, K. J. Måløy, and A. Aharony, Phys. Rev. Lett. 67, 584 (1991).

[15] M. Chaouche, N. Rakotomalala, D. Salin, B. Xu, and Y. C. Yortsos, Phys. Rev. E 49, 4133 (1994).

[16] T. A. Witten and L. M. Sander, Phys. Rev. Lett. 47, 1400 (1981).

[17] T. A. Witten and L. M. Sander, Phys. Rev. B 27, 5686 (1983).

[18] M. Eden, in Proceedings of the Fourth Berkeley Symposium on Mathematical Statistics and Probability, Vol. 4, edited by J. Neyman (University of California, Berkeley, 1961), pp. 223-239.
[19] H. P. Peters, D. Stauffer, H. P. Holters, and K. Loewenich, Z. Phys. B Con. Mat. 34, 399 (1979).

[20] M. J. Vold, J. Colloid Sci. 18, 684 (1963).

[21] D. N. Sutherland, J. Colloid Interface Sci. 22, 300 (1966).

[22] D. N. Sutherland, J. Colloid Interface Sci. 25, 373 (1967).

[23] P. Meakin, J. Colloid Interf. Sci. 96, 415 (1983).

[24] H. Mártin, J. Vannimenus, and J. P. Nadal, Phys. Rev. A 303205 (1984).

[25] D. Wilkinson and M. Barsony, J. Phys. A: Math. Gen. 17, L129 (1984).

[26] Y. Méheust, G. Løvoll, K. J. Måløy, and J. Schmittbuhl, Phys. Rev. E 66, 051603 (2002).

[27] P. Meakin, J. Feder, V. Frette, and T. Jøssang, Phys. Rev. A 46, 3357 (1992).

[28] J. Schmittbuhl, A. Hansen, H. Auradou, and K. J. Måløy, Phys. Rev. E 61, 3985 (2000).

[29] Z. Bo, D. Loggia, L. Xiaorong, G. Vasseur, and H. Ping, Eur. Phys. J. B 50, 631 (2006).

[30] R. Semer, J. A. Adams, and K. R. Reddy, Geotech. Geol. Eng. 16, 59 (1998).

[31] H. Geistlinger, G. Krauss, D. Lazik, and L. Luckner, Water Resource Res. 42, W07403 (2006).

[32] J. S. Selker, M. Niemet, N. G. McDuffie, S. M. Gorelick, and J.-Y. Parlange, Transp. Porous Med. 68, 107 (2007).

[33] X.-Z. Kong, W. Kinzelbach, and F. Stauffer, Chem. Eng. Sci. 64, 1528 (2009).

[34] X.-Z. Kong, W. Kinzelbach, and F. Stauffer, Chem. Eng. Sci. 65, 4652 (2010).

[35] W. Ji, A. Dahmani, D. P. Ahlfeld, J. D. Lin, and E. Hill, Ground Water Monit. Rem. 13, 115 (1993).

[36] G. Varas, V. Vidal, and J.-C. Géminard, Phys. Rev. E 83, 011302 (2011).

[37] G. Varas, G. Ramos, J.-C. Géminard, and V. Vidal, Front. Phys. 3, 44 (2015).

[38] J. W. Peterson, P. A. Lepczyk, and K. L. Lake, Environ. Geol. 38, 1 (1999).

[39] G. Varas, V. Vidal, and J.-C. Géminard, Phys. Rev. E 83, 061302 (2011).

[40] G. Varas, J.-C. Géminard, and V. Vidal, Gran. Matt. 15, 801 (2013).

[41] P. Philippe and M. Badiane, Phys. Rev. E 87, 042206 (2013). 